\title{
Heterogeneidade clínica e coexistência das neuropatias diabéticas: diferenças e semelhanças entre diabetes melito tipos 1 e 2
}

\author{
Clinical heterogeneity and coexistence of diabetic neuropathies: \\ difference and similarities between types 1 and 2 diabetes mellitus
}

Luiz Clemente Rolim', João Roberto de Sá', Antonio Roberto Chacra', Sérgio Atala Dib'

' Centro de Diabetes, Disciplina de Endocrinologia, Escola Paulista de Medicina (EPM), Universidade Federal de São Paulo (Unifesp), São Paulo, SP, Brasil
Correspondência para: Luiz Clemente Rolim

Rua Borges Lagoa, 1.065, conj. 110 04038-032 - São Paulo, SP, Brasil rolim777@gmail.com

Recebido em 23/Set/2008 Aceito em 14/Jul/2009

\section{RESUMO}

Objetivo: Estudar a heterogeneidade e a coexistência das neuropatias no diabetes melito tipos 1 (DMT1) e 2 (DMT2). Métodos: Foram avaliados 74 DMT2 e 20 DMT1 em relação à idade (anos), tempo de diagnóstico do DM (TDDM, em anos), índice de massa corpórea (IMC, $\mathrm{kg} / \mathrm{m}^{2}$ ), $\mathrm{HbA} 1 \mathrm{c}$ e tipo de neuropatia (critérios da American Diabetes Association). Resultados: DMT1 era mais jovem $(32,7 \pm 11$ versus $56,9 \pm 10,3 ; p=0,0001)$, com maior TDDM $(17,1 \pm 9,7$ versus 10,4 \pm $6,8 ; p=0,003)$ e menor IMC $(23,6 \pm 3,8$ versus $28,4 \pm 5,3 ; p=0,0005)$. A neuropatia autonômica cardiovascular (NAC) $(60 \%$ versus $32,4 \% ; p=0,02)$ e a coexistência desta com polineuropatia (PND) $(62,5 \%$ versus $33,3 \% ; p=0,03)$ foram mais prevalentes no DMT1; a PND dolorosa crônica (PNDDC) $(60,8 \%$ versus $30,0 \% ; p=0,009)$ o foi no DMT2. A HbA1c $(p=0,04)$ foi preditiva de PND em ambos os grupos. OTDDM $(p=0,03)$ e a PNDDC $(p=0,003)$ foram preditivos de NAC no DMT1. A idade $(p=0,0004)$ teve valor preditivo para PNDDC no DMT2. Conclusões: As neuropatias apresentam distribuição heterogênea no DMT1 e no DMT2. Com exceção do controle glicêmico, os fatores relacionados a essa complicação diferem de acordo com o tipo de diabetes. Arq Bras Endocrinol Metab. 2009;53(7):818-24.

Descritores

Diabetes melito; neuropatias diabéticas; diagnóstico; prevalência

\section{ABSTRACT}

Objective: To evaluate the heterogeneity and the coexistence of diabetic neuropathy (DNP) in type 1 (T1DM) and 2 (T2DM) diabetes mellitus. Methods: 74 T2DM and 20 T1DM patients were evaluated according to age (years), time from diagnosis of diabetes (TDD, years), body mass index (BMI, $\left.\mathrm{kg} / \mathrm{m}^{2}\right), \mathrm{HbA} 1 \mathrm{c}$ and DNP type (American Diabetes Association criteria). Results: T1DM was younger (32.7 \pm 11.0 versus $56.9 \pm 10.3 ; p=0.0001)$, leaner (BMI: $23.6 \pm 3.85$ versus $28.4 \pm 5.3 ; p=0.0005)$ and they had longerTDD (17.1 \pm 9.7 versus $10.4 \pm 6.8 ; p=0.003)$. Cardiovascular autonomic neuropathy (CAN) $(60 \%$ versus $32.4 \% ; p=0.02$ ) and its coexistence with polyneuropathy (PN) $(62.5 \%$ versus $33.3 \%$; $p=0.03)$ were more common inT1DM. Chronic painful polyneuropathy (CPP) was more prevalent in T2DM (60.8\% versus $30.0 \%$; $=0.009)$. Logistic regression showed $\mathrm{HbA} 1 \mathrm{c}$ as an independent variable related to PN $(p=0.04)$ in both groups. TDD $(p=0.03)$ and CPP $(p=0.003)$ were related to CAN in T1DM. Age $(p=0.0004)$ was related to CPP in T2DM. Conclusions: The DNP have shown a heterogeneity distribution in type 1 and type 2 diabetes mellitus. The related factors to different phenotypes of this complication, apart from hyperglycemia, may be variable between these two types of diabetes mellitus. Arq Bras Endocrinol Metab. 2009;53(7):818-24.

Keywords

Diabetes mellitus; diabetic neuropathies; diagnosis; prevalence 


\section{INTRODUÇÃO}

A s neuropatias diabéticas (ND) constituem a complicação crônica mais frequente do diabetes melito $(\mathrm{DM})$ e a primeira causa de amputação não traumática de membros inferiores em adultos, embora a maioria dos casos $(50 \%$ a $75 \%)$ permaneça subdiagnosticada e assintomática (1-3). Como as ND compreendem várias síndromes clínicas com diferentes mecanismos patogenéticos, o diagnóstico diferencial entre estas é importante, do ponto de vista terapêutico e prognóstico.

A heterogeneidade clínica das ND é conhecida há aproximadamente um século (4), porém, ainda há controvérsias na literatura em relação às diferenças no seu fenótipo clínico entre os dois tipos principais de DM: o 1 (DMT1) e o 2 (DMT2).

A deficiência de insulina grave, que é característica do DMTl e que pode ocorrer em fases mais tardias do DMT2, desempenha um papel importante na fisiopatologia das ND, interagindo com a predisposição genética e a deterioração de fatores neurotróficos (5). Em uma análise inicial, seria possível inferir que a fisiopatologia multifatorial desse tipo de microangiopatia diabética resultaria em diferenças em sua apresentação clínica, gravidade e evolução entre os dois tipos principais de DM. Entretanto, estudos populacionais e prospectivos demonstram diferenças modestas nesse sentido. O estudo mais abrangente, até o momento, foi o Rochester Diabetic Neuropathy Study (6), que avaliou a prevalência de ND em DMT2 e DMTl e encontrou diferenças apenas em relação à gravidade dos dois tipos: a prevalência de polineuropatia diabética (PND) grave foi maior no DMTl $(6 \%)$ do que no DMT2 (1\%). A distribuição de frequência por tipo de neuropatia foi semelhante no DMT1 e no DMT2, inclusive a proporção de casos de PND sintomática (15\% versus $13 \%)$ e de casos de síndrome do túnel do carpo (STC) (33\% versus $35 \%)$, respectivamente nestes dois tipos de diabetes.

No presente estudo, o termo "polineuropatia diabética”, caracterizado pela degeneração distal retrógrada e simétrica dos nervos sensitivos e ou motores (3), é utilizado como sinônimo de neuropatia periférica sensitivomotora distal. Pelo menos 50\% dos indivíduos com DM e PND apresentam também algum grau de disfunção autonômica $(2,7)$. Aqueles pacientes com predominância dos sinais e sintomas desta última são classificados como portadores de neuropatia autonômica diabética (NAD). Já a neuropatia autonômica cardiovascular (NAC) ocorre quando há lesão das fibras autonômicas periféricas (simpático e parassimpático) relacionadas ao sistema cardiovascular (SCV), resultando em distúrbios na sua regulação neuro-humoral (7).

A relação entre o acometimento somático e a NAC durante a história natural da PND permanece em discussão; existem, porém, estudos que mostram que a maior parte dos pacientes com PND apresenta algum grau de disautonomia cardiovascular e vice-versa (7-10). A NAD apresenta um caráter progressivo em relação aos sistemas comprometidos, geralmente iniciando-se pelos sistemas neurovascular cutâneo (microcirculação dos pés) e cardiovascular, acometendo, posteriormente, o sistema gastrintestinal e geniturinário.

Em recente revisão (7), ressaltou-se que a NAC constitui uma das complicações de maior repercussão clínica no DM, mas, ao mesmo tempo, está entre as menos diagnosticadas. A NAC está associada a uma mortalidade por evento cardiovascular duas a três vezes maior e a uma mortalidade total até cinco vezes maior em relação aos portadores de DM sem disautonomia.

Como salientado, existem vários estudos que mostram a correlação entre a lesão progressiva das fibras somáticas (sensitivas e motoras) e autonômicas (9-11). No entanto, há controvérsias também sobre a existência de diferença, nesse sentido, entre os dois principais tipos de DM.

O objetivo do presente trabalho foi estudar a prevalência e a heterogeneidade dos diferentes fenótipos clínicos de ND entre pacientes portadores de DMTl e DMT2, bem como avaliar e comparar a coexistência de PND e NAC entre esses indivíduos.

\section{INDIVÍDUOS E MÉTODOS}

\section{Indivíduos}

Foram avaliados cem indivíduos consecutivos, de ambos os sexos, portadores de DM de acordo com os critérios da American Diabetes Association (ADA) (12) e encaminhados por endocrinologistas ao Setor de Neuropatias Diabéticas do Centro de Diabetes da Escola Paulista de Medicina (EPM) da Universidade Federal de São Paulo (Unifesp), no período de fevereiro de 2004 a dezembro de 2005. O presente estudo foi aprovado pelo Comitê de Ética da Unifesp-EPM (número 1011/03), e os participantes assinaram um termo de consentimento livre e esclarecido antes do início da avaliação.

\section{Métodos}

Os indivíduos foram submetidos a uma avaliação clínica geral composta por: história clínica, idade, tempo de 
diagnóstico do diabetes melito (TDDM), altura $(\mathrm{m})$, peso $(\mathrm{kg})$, índice de massa corpórea $\left(\mathrm{IMC}, \mathrm{kg} / \mathrm{m}^{2}\right)$ e pressão arterial $(\mathrm{mmHg})$.

Foram critérios para exclusão: presença de outro fator etiológico, diferente do DM, para polineuropatia; sinais clínicos ou laboratoriais de insuficiência hepática, renal, cardíaca ou anemia e neoplasias; uso atual ou pregresso de droga potencialmente neurotóxica; episódio de hipoglicemia grave (glicemia $<40 \mathrm{mg} / \mathrm{dL}$ ) com perda de consciência ou convulsão nos últimos seis meses.

\section{Exame neurológico}

No presente estudo, foi realizada uma visão sistemática do exame neurológico geral. São dados de interesse para o estudo:

- sensibilidade:

a) térmica (tubos de ensaio com água a $0^{\circ} \mathrm{C}$ e $45^{\circ} \mathrm{C}$ ) verificados com termômetro de imersão digital e aplicados no dorso do polegar e do hálux, bilateralmente;

b) dolorosa (estilete de madeira): dorso do hálux e do polegar, bilateralmente;

c) tátil (monofilamento de Semmes-Weinstein, 10 $\mathrm{g}$ ): polpas do hálux, do primeiro, terceiro e quinto metatarsos, bilateralmente;

d) vibratória (teste sensorial quantitativo): obtevese o limiar de percepção vibratória por meio de um biotesiômetro (Biomedical Instruments, Newbury, Ohio, USA) graduado em uma escala de 0 a $50 \mathrm{~V}$. Aplicou-se o dispositivo vibratório às extremidades do hálux e do polegar, bilateralmente, e a voltagem foi gradualmente aumentada até o paciente sentir o estímulo inicial (limiar). Desprezou-se a primeira leitura; o valor final considerado foi a média dos valores obtidos em três medidas. Considerou-se como valor normal para o hálux um limiar de até $8 \mathrm{~V}$. Para a ponta do polegar, considerou-se normal um limiar até $6 \mathrm{~V}$. O objetivo desse exame foi avaliar quantitativamente a função das fibras A (13).

- reflexos da musculatura esquelética:

a) membros superiores: bicipital, tricipital e estilo radial;

b) membros inferiores: patelar (quadríceps), Aquileu - manobra de Jendrassik - e o cutâneo plantar, para pesquisa do sinal de Babinski.

Os reflexos musculares foram pesquisados com um martelo neurológico padrão e o paciente, relaxado. Os reflexos foram classificados de acordo com a seguinte escala: 0 : ausente; 1 : presente.
- força muscular e marcha: manobra de Mingazini, para avaliar a força dos quadríceps e a presença de deficiência proximal ou assimétrica. A marcha (normal, sobre o antepé e sobre os calcanhares) foi examinada com a finalidade de avaliar a deficiência motora distal e a gravidade da PND (13);

- sinais de Phalen (manobra da prece durante um minuto) e de Tinel (percussão digital do nervo mediano no túnel do carpo). Os sinais são considerados positivos quando o paciente relata acroparestesias (Phalen) e dor ou choque (Tinel) na distribuição do nervo mediano.

\section{Testes autonômicos}

Para o diagnóstico de NAC, utilizou-se o método de Ewing, que inclui os três testes de variabilidade da frequência cardíaca preconizados pela $\operatorname{ADA}(7,9)$ : a relação expiração/inspiração $(\mathrm{E} / \mathrm{I})$, a resposta à manobra de Valsalva e a alteração postural (teste ortostático ou razão 30:15).

Os pacientes foram orientados a comparecer em jejum de oito horas e evitar o uso de antidepressivos, neurolépticos, cafeína, nicotina, anti-histamínicos, anti-hipertensivos pelo menos 12 horas antes do exame. A relação E/I foi realizada por meio de uma respiração única de dez segundos (cinco segundos para cada fase) e calculada como o maior intervalo R-R durante a expiração dividido pelo menor intervalo $\mathrm{R}-\mathrm{R}$ durante a inspiração. A relação na mudança postural $(30: 15)$ foi a razão entre o mais longo intervalo R-R durante 20 a 40 batimentos em pé e o mais curto intervalo R-R durante os batimentos de 5 a 25 em pé. Para a avaliação da resposta da frequência cardíaca durante a manobra de Valsalva, a relação foi do maior intervalo R-R (obtido na fase de repouso após os 15 segundos de expiração forçada) pelo menor intervalo R-R (obtido durante a exalação forçada, por 15 segundos, em dispositivo bucal mantendo um manômetro em $40 \mathrm{mmHg}$ ) (7). As relações foram consideradas anormais quando estavam abaixo do quinto percentil de população normal pareada por idade (14-16).

\section{Critérios diagnósticos}

O diagnóstico da PND teve como base os consensos de San Antonio (Texas, EUA) $(13,14)$ e da ADA (15). Assim, a PND foi definida pela presença de pelo menos duas alterações (simétrica e distal) no exame neurológico específico descrito. 
A presença de NAC foi diagnosticada quando pelo menos dois dos três testes descritos estavam alterados. Em casos duvidosos ou limítrofes, os testes foram repetidos em uma segunda ocasião.

A STC foi diagnosticada com base em critérios clínicos: nos sinais de Tinel (sensibilidade de 61\%), de Phalen (sensibilidade de 46\%), na presença de hipotrofia da eminência tenar e no sinal do fletir do punho (flick sign), quando o paciente relata acordar com a mão formigando e rapidamente melhora, ao supinar e pronar rapidamente o punho (17-19).

Para os diagnósticos de PND dolorosa crônica (PNDDC), da PND indolor com perda sensitiva parcial ou completa (PNDI) e das mononeuropatias cranianas e tronculares, foram utilizados os critérios clínicos (história e exame físico) da ADA (17). Os diagnósticos de amiotrofia diabética e de polineuropatia desmielinizante inflamatória crônica (PNPDIC) foram realizados com base nos critérios da American Academy of Neurology (AAN) (19) e, para tal, os pacientes foram submetidos aos exames de eletroneuromiografia (EMG) e liquor.

\section{Classificação}

A divisão das ND teve como base a classificação do consenso da ADA em 2005 (15), com duas modificações:

- a PND foi subdividida em dois estágios (PNDDC e PNDI), conforme os critérios da revisão técnica da ADA em 2004 (17);

- foi acrescentado um terceiro grupo, além das PND e das neuropatias focais e multifocais: as formas mistas, caracterizadas pela associação de uma PND com uma neuropatia focal ou multifocal $(18,19)$.

\section{Avaliação do controle glicêmico}

O controle glicêmico foi avaliado pela média das hemoglobinas glicadas (HbAlc, método HPLC - v.r: 3,6 a $5,3 \%)$ nos dois últimos anos, obtidas no prontuário dos pacientes.

\section{Análise estatística}

As variáveis com distribuição normal foram relatadas como porcentagens ou médias \pm desvio-padrão. Quando estas não apresentavam distribuição normal, utilizou-se a transformação logarítmica. Variáveis numéricas de interesse foram comparadas pelo teste $t$ de Student, para duas amostras independentes. Em se tratando de variáveis categóricas ( 0 ou 1 ), empregou-se o teste do $\chi^{2}$ ou exato de Fisher. Para testar a associação entre duas variáveis, utilizou-se o coeficiente de correlação (Pearson ou Spearman, conforme a necessidade). Utilizou-se ANOVA para comparar os grupos (DMTl e DMT2). Para identificar os previsores independentes de NAC, PND, STC e PDDC, utilizou-se a análise de regressão logística múltipla (stepwise), com $\mathrm{r}^{2}<0,05$. Fixou-se o nível de significância em $\mathrm{p}<0,05$ e utilizou-se o software SigmaStat (versão 3.1 para Windows, USA, 2004) para todas as análises.

\section{RESULTADOS}

Entre os cem indivíduos com DM consecutivamente encaminhados para avaliação no Setor de Neuropatias do Centro de Diabetes, 94 foram avaliados no protocolo, pois seis estavam dentro dos critérios de exclusão do estudo. O grupo estudado foi composto, então, de 20/94 $(21,3 \%)$ com DMTl e de 74/94 (78,7\%) com DMT2.

$\mathrm{Na}$ tabela 1 , estão representados os valores médios de idade, TDDM, altura, gênero, IMC e HbAlc em ambos os grupos. Não houve diferença estatística entre os dois grupos em relação a altura, gênero e HbAlc. A faixa etária era superior e o IMC, maior, como esperado, no Grupo DMT2, enquanto o TDDM foi maior no Grupo DMTl.

As distribuições por frequência dos diversos fenótipos clínicos das ND encontradas nos dois grupos estão representadas na tabela 2. A prevalência de NAC foi duas vezes maior no DMTl do que no DMT2 (60\% versus $32,4 \% ; \mathrm{p}=0,024)$. No DMTl, houve seis vezes mais casos de PNDI ( $50 \%$ versus $8,1 \% ; \mathrm{p}=0,0001)$ e quase duas vezes mais casos de NAC coexistindo com PNDDC $(62,5 \%$ versus $33,3 \% ; \mathrm{p}=0,034)$. As prevalências de formas mistas de ND foram semelhantes entre os dois tipos de DM.

Os dados da análise de regressão logística estão resumidos na tabela 3 , na qual se verifica que a HbAlc permaneceu como a única variável independente com valor preditivo significativo para PND em ambos os grupos. Enquanto PNDDC e TDDM permaneceram como variáveis independentes e significantes relacionadas à presença de NAC no Grupo DMTl, apenas o valor de HbAlc permaneceu significante para prever NAC no grupo DMT2. Por outro lado, somente a existência de NAC apresentou valor preditivo para PNDDC no DMT1, enquanto, no DMT2, permaneceram como preditores a idade e a $\mathrm{HbAlc}$, mostrando uma relação recíproca entre NAC e PNDDC apenas no grupo DMTl. 
Tabela 1. Características demográficas e metabólicas dos indivíduos estudados $(n=94)$, estratificados segundo 0 tipo de diabetes*

\begin{tabular}{lccc}
\hline & DMT1 $(\mathbf{n}=\mathbf{2 0})$ & DMT2 $(\mathbf{n}=\mathbf{7 4})$ & Valor de p \\
\hline Idade (anos) & $32,7 \pm 11$ & $56,9 \pm 10,3$ & 0,0001 \\
Altura $(\mathrm{m})$ & $1,62 \pm 0,14$ & $1,63 \pm 0,11$ & 0,63 \\
IMC $\left(\mathrm{kg} / \mathrm{m}^{2}\right)$ & $23,6 \pm 3,8$ & $28,4 \pm 5,3$ & 0,0005 \\
TDDM (anos) & $17,1 \pm 9,7$ & $10,4 \pm 6,8$ & 0,003 \\
Gênero (\%F) & 55 & 50 & 0,69 \\
HDA1C (\%) & $9,2 \pm 2,2$ & $8,4 \pm 2,1$ & 0,25 \\
\hline
\end{tabular}

* Dados expressos em média \pm desvio-padrão ou em porcentagens.

TDDM: tempo de diagnóstico do diabetes melito; HbA1c: hemoglobina glicada; IMC: índice de massa corpórea. F: feminino.

Tabela 2. Prevalência e distribuição das neuropatias diabéticas (ND) nos indivíduos estudados de acordo com o tipo de diabetes melito*

\begin{tabular}{|c|c|c|c|}
\hline $\begin{array}{l}\text { Tipo de neuropatia } \\
\text { diabética }\end{array}$ & $\begin{array}{c}\text { DMT1 }(n=20) \\
(\%)\end{array}$ & $\begin{array}{c}\text { DMT2 }(n=74) \\
(\%)\end{array}$ & $\underset{\mathbf{p}}{\text { Valor de }}$ \\
\hline NAC & $12(60)$ & $24(32,4)$ & 0,024 \\
\hline PND (PNDDC + PNDI) & $16(80)$ & $51(68,9)$ & 0,29 \\
\hline $\begin{array}{l}\text { PND dolorosa crônica } \\
\text { (PNDDC) }\end{array}$ & $6(30)$ & $45(60,8)$ & 0,009 \\
\hline PND indolor (PNDI) & $10(50)$ & $6(8,1)$ & 0,0001 \\
\hline MNP craniana & $0(0)$ & $3(4,1)$ & 0,077 \\
\hline $\begin{array}{l}\text { Radiculoneuropatia } \\
\text { toracolombar }\end{array}$ & $1(5)$ & $3(4,1)$ & 0,86 \\
\hline STC & $4(20)$ & $14(18,6)$ & 0,91 \\
\hline PNDIC & $1(5)$ & $1(1,3)$ & 0,47 \\
\hline Amiotrofia diabética & $0(0)$ & $3(4,1)$ & 0,077 \\
\hline$N A C+P N D$ & $10 / 16(62,5)$ & 17 / $51(33,3)$ & 0,034 \\
\hline Formas mistas & $5(25)$ & $18(24,3)$ & 0,95 \\
\hline
\end{tabular}

* Dados expressos em números de indivíduos e porcentagens.

NAC: neuropatia autonômica cardiovascular; PND: polineuropatia diabética; MNP: mononeuropatia; STC: síndrome do túnel do carpo; PNDIC: polineuropatia desmielinizante inflamatória crônica.

Tabela 3. Regressão logística múltipla (stepwise) entre variáveis dependentes (NAC, PND e PNDDC) e as respectivas variáveis independentes que permaneceram significantes no modelo, nos portadores de diabetes melito do tipo 1 e 2 estudados

\begin{tabular}{|c|c|c|c|c|}
\hline \multirow[b]{2}{*}{$\begin{array}{l}\text { Variável } \\
\text { dependente }\end{array}$} & \multicolumn{4}{|c|}{ Variáveis independentes e significantes $\left(r^{2}<0,05\right)$} \\
\hline & DMT1 (n = 20) & Valor de $\mathbf{p}$ & DMT2 $(n=74)$ & Valor de $\mathbf{p}$ \\
\hline \multirow{2}{*}{ NAC } & TDDM & 0,03 & \multirow{2}{*}{$\mathrm{HbA1c}$} & \multirow{2}{*}{0,04} \\
\hline & PNDDC & 0,003 & & \\
\hline PND & $\mathrm{HbA1c}$ & 0,04 & $\mathrm{HbA1C}$ & 0,04 \\
\hline PNDDC & NAC & 0,019 & Idade & 0,0004 \\
\hline
\end{tabular}

NAC: neuropatia autonômica cardiovascular; PND: polineuropatia diabética; PNDDC: PND dolorosa crônica; TDDM: tempo de diagnóstico do diabetes melito; HbA1c: hemoglobina glicada.

\section{DISCUSSÃO}

O presente estudo avaliou a prevalência e a distribuição dos diferentes subtipos clínicos de ND entre indivíduos com DMT1 e DMT2 encaminhados por endocrinologistas. Uma distribuição heterogênea dos diferentes tipos de ND entre os dois tipos de diabetes foi observada. A PNDI e a NAC foram mais frequentes no DMTI e a PNDDC, no DMT2. Os dois grupos avaliados apresentavam o mesmo e insatisfatório grau de controle glicêmico por ocasião do estudo.

A análise de regressão logística no Grupo DMTl mostrou que o TDDM e a presença de PNDDC foram fatores independentes e significantes para a existência de NAC. Por outro lado, no grupo DMT2, somente a $\mathrm{HbAlc}$ foi fator independente e significante para a presença de NAC e apenas a idade, para a PNDDC.

$\mathrm{O}$ fato de ambos os grupos terem o mesmo grau de controle glicêmico (HbAlc > 8\%) e apresentarem diferenças na heterogeneidade clínica das ND sugere que, além da hiperglicemia, alterações inerentes à etiopatogenia do tipo de diabetes (autoimunidade e deficiência de insulina no DMTl, e deficiência e resistência à ação da insulina no DMT2) podem colaborar, por meio de mecanismos diferentes, para o desenvolvimento dessa complicação microangiopática $(2,20,21)$.

Existem evidências de que a patogênese da PND é multifatorial, mas a teoria mais aceita, no momento, é a da hiperglicemia crônica como um fator primário (22). Estudos experimentais mostram que a hiperglicemia persistente (relacionada à deficiência de insulina e por meio da glicotoxicidade) pode gerar alterações metabólicas e vasculares precoces nos componentes neurais $(23,24)$. No nosso estudo, a HbAlc foi um fator preditivo significante para a PNDDC, tanto no DMTl como no DMT2.

Contudo, parece improvável que os fatores metabólicos concorram igualmente para todos os pacientes com neuropatia. Outros fatores, como idade, hipertensão arterial, tabagismo, obesidade e hipertrigliceridemia, podem acelerar os processos patológicos iniciados pela hiperglicemia, como demonstrado em estudos prospectivos tanto no DMT2 (1) como no DMTl (25).

A maior prevalência de PNDI no grupo DMTl pode sugerir estádios mais avançados e maior gravidade da PND nesses pacientes. Esse achado tem suporte no principal estudo prospectivo (6) já publicado nesse sentido, que encontrou diferenças entre o DMTl e o DMT2 em relação à gravidade da PND. A prevalência de PND avançada foi maior no DMTl (6\%) do que no DMT2 (1\%). Há dez anos, um estudo prospectivo relatava o próprio DMTl como um fator de risco para PND grave (26). 
Um fato que deve ser destacado no presente estudo é o de que a análise de regressão múltipla mostrou uma relação recíproca entre PNDDC e a NAC somente no DMTl. Esse achado está de acordo com alguns estudos (27-29), segundo os quais a NAC está frequentemente presente em pacientes com PNDDC no DMTl. Uma explicação possível, nessa direção, poderia ser um maior comprometimento das fibras $\mathrm{C}$ (dor, sensibilidade ao calor e fibras autonômicas) nestes pacientes e/ou relacionado ao maior TDDM (27), que é um fator de risco bem determinado tanto para a PNDDC como para a NAC (29-31).

Por outro lado, no DMT2, parece haver uma divergência quanto ao desenvolvimento e à ocorrência mútua de PND e NAC. Em um estudo prospectivo de dez anos (31), a piora da disautonomia cardiovascular (NAC) ocorreu independentemente da piora nos outros parâmetros neurofisiológicos. Neste estudo, os autores também encontraram diferenças entre os fatores preditivos para o desenvolvimento de NAC ou de PND no DMT2. Por exemplo, enquanto a hipoinsulinemia (fases mais tardias do DMT2) estava relacionada à PND, a hiperinsulinemia (que pode preceder o DMT2 clínico) relacionava-se à NAC, embora a hiperglicemia tenha contribuído igualmente ao desenvolvimento das duas. Esses dados são coerentes com outros estudos, nos quais a PND de fibras $\mathrm{C}$ (amielínicas) precede a PND de fibras A (mielínicas) na história natural da PND no DMT2 $(28,29)$.

Como no DMTl a insulinopenia ocorre desde o diagnóstico e a resistência insulínica (induzida pela glicotoxicidade) é mais tardia, espera-se um desenvolvimento mais lento e retardado da NAC. No entanto, quando esta se torna sintomática, sua coexistência com a PND é elevada. De fato, na presente casuística de DMTl, encontrou-se uma coexistência de $62,5 \%$ (portadores de PND que também tinham NAC), fato que está de acordo com outros estudos, nos quais essa coexistência variou entre $62,9 \%$ e $72,9 \%(10,32)$.

Outra manifestação da PND é a STC. No presente estudo, a presença de STC foi semelhante entre os dois grupos. No Rochester Diabetic Neuropathy Study (6), estudo prospectivo e populacional, também não houve diferença significante em relação à prevalência de STC entre os pacientes com DMTl e DMT2; houve, inclusive, uma correlação positiva e significante entre STC (diagnosticada por eletromiografia) e PND. Deve-se considerar, entretanto, a possibilidade de que, na presente casuística, a prevalência de STC esteja subestimada, pois, ao contrário do estudo de Rochester, o pre- sente diagnóstico foi apenas clínico - sem a avaliação sistemática das medidas de condução nervosa.

Em relação à radiculoneuropatia toracolombar, embora seja uma condição rara, não foi encontrada diferença entre a sua prevalência nos grupos estudados. $\mathrm{O}$ mesmo foi relatado por um trabalho prospectivo (20) com um número maior de pacientes. Os quatro casos de radiculoneuropatia toracolombar da nossa casuística apresentavam NAC concomitantemente, sugerindo sobreposição de mecanismos patogenéticos para essas duas últimas. Nesse sentido, há também a hipótese de que a radiculopatia toracolombar e a amiotrofia diabética possuem mecanismos etiopatogênicos comuns, mais exatamente infartos acometendo nervos, raízes e plexos (radiculoplexoneuropatia), secundários a uma vasculite $(17,33)$.

Entre as limitações do presente estudo estão: a impossibilidade de utilizar a eletromiografia como instrumento diagnóstico para a STC, como ocorreu em relatos semelhantes (34); a ausência de avaliação da relação dos tipos de ND com marcadores da retino e nefropatia diabéticas, já que estas pertencem ao mesmo grupo de complicações crônicas do DM (microangiopatia); por se tratar de um estudo transversal, com uma amostra relativamente pequena $(\mathrm{n}=100)$ e não aleatória, não é possível generalizar os resultados para o universo do DMT1 ou do DMT2.

Em conclusão, em um grupo de pacientes com DMT1 e DMT2, com controle glicêmico insatisfatório e encaminhado com suspeita de ND, a prevalência dos subtipos clínicos dessa complicação crônica da doença foi heterogênea. A NAC e a PNDI foram mais prevalentes no DMTl e a PNDDC, no DMT2.

Enquanto o valor de $\mathrm{HbAlc}$ teve valor preditivo significante para PND em ambos os tipos de DM, os fatores relacionados à presença das duas neuropatias mais prevalentes (PNDDC e NAC) foram diferentes. A PNDDC se relacionou à idade cronológica no DMT2 e à presença de NAC no DMTl. Por outro lado, a NAC se relacionou ao TDDM no DMTl e ao valor de HbAlc no DMT2.

As condições do estudo (DMTl com TDDM superior a 15 anos e controle glicêmico insatisfatório, HbAlc $\geq 8,5 \%$ ) ressaltam a alta prevalência da NAC, que é uma complicação muitas vezes subdiagnosticada nos portadores de DMTl, além de ser um reconhecido marcador de risco para macroangiopatia e de mau prognóstico para nefropatia nesses indivíduos (7).

A importância da pesquisa da NAC no DM deve ser ressaltada e, entre os dois principais tipos de diabetes, 
as diferenças nos fatores que concorrem para o desenvolvimento da mesma devem ser consideradas quando da elaboração de estudos de prevenção, diagnóstico e tratamento dessa condição.

Agradecimentos: à Professora Doutora Ieda Terezinha Verreschi, pelas críticas e sugestões.

Declaração: os autores declaram não haver conflitos de interesse científico neste estudo.

\section{REFERÊNCIAS}

1. Partanen J, Niskanen L, Lehtinen J, Mervaala E, Siitonen O, Uusitupa M. Natural history of peripheral neuropathy in patients with non insulin dependent diabetes mellitus. N Engl J Med. 1995;333(2):89-94.

2. Watkins PJ, Thomas PK. Diabetes mellitus and the nervous system. J Neurol Neurosurg Psychiatry. 1998;65(5):620-32.

3. Ropper AH, Brown RH. Diseases of the peripheral nerves. In: Adams \& Victor's Principles of Neurology. 8th ed. NewYork: McGrawHill; 2005. p. 1110-77.

4. Auche MB. Des alterations des nerfs périphériques chez les diabetiques. Arch Med Exp Anat Pathol. 1890;2:635-76.

5. Harati Y. Diabetic neuropathies: unanswered questions. Neurol Clin. 2007;25(1):303-17.

6. Dyck PJ, Kratz KM, Karnes JL, Litchy WJ, Klein R, Pach JM. The prevalence by staged severity of various types of diabetic neuropathy, retinopathy, and nephropathy in a population-based cohort: the Rochester Diabetic Neuropathy Study. Neurology. 1993;43(4):817-24.

7. Rolim LCSP, Sá JR, Chacra AR, Dib AS. Diabetic cardiovascular autonomic neuropathy: risk factors, clinical impact and early diagnosis. Arq Bras Cardiol. 2008;90(4):e23-e31.

8. Low PA, Vernino S, Suarez G. Autonomic dysfunction in peripheral nerve disease. Muscle Nerve. 2003;27(6):646-61.

9. Vinik Al, Ziegler D. Diabetic cardiovascular autonomic neuropathy. Circulation. 2007;115(3):387-97.

10. Tackmann W, Kaeser HE, Berger W, Rüeger AN, Violier E. Autonomic disturbances in relation to sensorimotor peripheral neuropathy in diabetes mellitus. J Neurol. 1981;224(4):273-81.

11. Veves A, Young MJ, Manes C, Boulton AJ. Differences in peripheral and autonomic nerve function measurements in painful and painless neuropathy. A clinical study. Diabetes Care. 1994;17(10):1200-2.

12. Genuth $S$, Alberti KG, Bennett $P$, Buse J, Defronzo R, Kahn R, Kitzmiller J, Knowler WC, Lebovitz H, Lernmark A, Nathan D, Palmer J, Rizza R, Saudek C, Shaw J, Steffes M, Stern M, Tuomilehto J, Zimmet P; Expert Committee on the Diagnosis and Classification of Diabetes Mellitus. Follow-up report on the diagnosis of diabetes mellitus. Diabetes Care. 2003;26(11):3160-7.

13. Consensus statement: Report and recommendations of the San Antonio conference on diabetic neuropathy. American Diabetes Association, American Academy of Neurology. Diabetes Care. 1988;11(7):592-7.

14. Kahn R. Proceedings of a consensus development conference on standardized measures in diabetic neuropathy. Autonomic nervous system testing. Diabetes Care. 1992;15(8):1095-103.
15. Boulton AJ, Vinik Al, Arezzo JC, Bril V, Feldman EL, Freeman R, et al. Diabetic neuropathies. Diabetes Care. 2005;28(4):956-62.

16. Vinik Al, Maser RE, Mitchell BD, Freeman R. Diabetic autonomic neuropathy. Diabetes Care. 2003;26(5):1553-79.

17. Boulton AJ, Malik RA, Arezzo JC and Sosenko JM. Diabetic somatic neuropathies. Diabetes Care. 2004;27(6):1458-86.

18. Thomas PK. Metabolic neuropathy. J R Coll Physicians Lon. 1973;7(2):154-60.

19. Llewelyn JG, Tomlinson DR, Thomas PK. Diabetic neuropathies. In: Dyck PJ, Thomas PK, editors. Peripheral neuropathy. 4th Ed. Philadelphia: Elsevier Saunders; 2005. v. 2. p. 1951-92.

20. O'Hare JA, Abuaisha F, Geoghegan M. Prevalence and forms of neuropathic morbidity in 800 diabetics. Ir J Med Sci. 1994;163(3):132-5.

21. Sima AAF, Kamiya H. Diabetic neuropathy differs in type 1 and type 2 diabetes. Ann NY Ac Sci. 2006;1084:235-49.

22. Brownlee M. The pathobiology of diabetic complications: a unifying mechanism. Diabetes. 2005;54(6):1615-25.

23. Cameron NE, Cotter MA, Low PA. Nerve blood flow in early experimental diabetes in rats: relation to conduction deficits. Am J Physiol Endocrinol Metab. 1991;261(1 Pt 1):E1-8.

24. The effect of intensive diabetes therapy on the development and progression of neuropathy. The Diabetes Control and Complications Trial Research Group. Ann Intern Med. 1995;122(8):561-8.

25. Tesfaye S, Chaturvedi N, Eaton SE, Ward JD, Manes C, lonescuTirgoviste C, Witte DR, Fuller JH; EURODIAB Prospective Complications Study Group. Vascular risk factors and diabetic neuropathy. N Engl J Med. 2005;352(4):341-50.

26. Dyck PJ, Davies JL, Wilson DM, Service FJ, Melton LJ 3rd, O'Brien PC. Risk factors for severity of diabetic polineuropathy: intensive longitudinal assessment of the Rochester Diabetic Neuropathy Study cohort. Diabetes Care. 1999;22(9):1479-86.

27. Tsigos $C$, Reed P, Weinkove C, White A, Young RJ. Plasma norepinephrine in sensory diabetic polyneuropathy. Diabetes Care. 1993;16(5):722-7.

28. Young RJ, Zhou YQ, Rodriguez E, Prescott RJ, Ewing DJ, Clarke $B F$. Variable relationship between peripheral somatic and autonomic neuropathy in patients with different syndromes of diabetic polyneuropathy. Diabetes. 1986;35(2):192-7.

29. Lanting P, Faes TJC, Bertelsmann FW, Heimans JJ, van der Veen EA. Autonomic and somatic peripheral nerve function and the correlation with neuropathic pain in diabetic patients. J Neurol Sci. 1989;94(1-3):307-17.

30. Young RJ, Macintyre CC, Martyn CN, Prescott RJ, Ewing DJ, Smith $A F$, et al. Progression of subclinical polineuropathy in young patients with type 1 diabetes: association with glycemic control and microangiopathy. Diabetologia. 1986;29(3):156-61.

31. Töyry JP, Partanen JV, Niskanen LK, Länsimies EA, Uusitupa MI. Divergent development of autonomic and peripheral somatic neuropathies in NIDDM. Diabetologia. 1997;40(8):953-8.

32. Lluch I, Hernández A, Real JT, Morillas C, Tenés S, Sánchez C, et al. Cardiovascular autonomic neuropathy in type 1 diabetic patients with and without peripheral neuropathy. Diabetes Res Clin Pract. 1998;42(1):35-40.

33. Dyck PJB, Norell JE, Dyck PJ. Microvasculitis and ischemia in diabetic lumbosacral radiculoplexus neuropathy. Neurology. 1999;53(9):2113-21.

34. Green DA, Brown MJ, Braunstein SN, Schwartz SS, Ashbury AK, Winegrad Al. Comparison of clinical course and sequential electrophysiological tests in diabetics with symptomatic polyneuropathy and its implications for clinical trials. Diabetes. 1981;30(2):139-47. 